\title{
Collaborative Technologies for Knowledge Management: Making the Tacit Explicit?
}

\author{
Vicki Romaldi \\ University of South Australia, Adelaide, Australia
}

\author{
vicki.romaldi@unisa.edu.au
}

\begin{abstract}
The theory of organisational knowledge creation indicates the distinction between tacit and explicit knowledge is extremely important in corporate knowledge management efforts. Additionally, in this context, the sharing of tacit knowledge is considered to be a critical component of successful knowledge management initiatives. This paper explores the value of using collaborative technologies as enablers of knowledge management. It primarily focuses on the conversion of tacit knowledge to explicit knowledge through the use of technologies with hyperlinking and hypermedia capabilities. Organisational issues surrounding the capture of tacit knowledge are also introduced by discussing the essential ingredients of successful knowledge management programs.
\end{abstract}

Keywords: knowledge management, tacit knowledge, hyperlinking, hypermedia, organisational culture.

\section{Introduction}

For some time now, the management of knowledge has been at the corporate centre stage. In consideration of its inherent and potential value, organisations continue to actively explore how to capitalise on its worth by investing widely in the implementation of enabling strategies and technology. With such endeavours in mind, the theory of organisational knowledge creation suggests the distinction between tacit and explicit knowledge is extremely important, and the sharing of tacit knowledge is a critical component of successful organisational knowledge management programs. Tacit knowledge is therefore considered to be an essential target of many knowledge management initiatives, and according to Delphi (1997, p. 13) this is "because it is the repository of the organisation's most strategically valuable knowledge."

Wiig (1997, p. 1) indicates that although "no general approach to managing knowledge has been commonly accepted" a number of notions continue to be advanced. These include management of explicit knowledge via technology-based networks, the management of intellectual capital (structural and human), and the management of knowledge in a broader sense encapsulating aspects relating to the previous two notions.

This paper focuses on knowledge management in the broader sense. It explores the value of collaborative technologies as enablers of knowledge management and primarily focuses on the conversion of tacit

Material published as part of these proceedings, either on-line or in print, is copyrighted by Informing Science. Permission to make digital or paper copy of part or all of these works for personal or classroom use is granted without fee provided that the copies are not made or distributed for profit or commercial advantage AND that copies 1) bear this notice in full and 2) give the full citation on the first page. It is permissible to abstract these works so long as credit is given. To copy in all other cases or to republish or to post on a server or to redistribute to lists requires specific permission

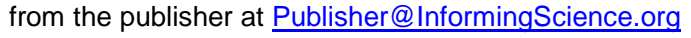

knowledge to explicit knowledge for use by the body corporate. Following background discussion on knowledge management, knowledge management tools and technologies are explored. Collaborative technologies with hyperlinking and hypermedia capabilities are introduced as enablers of the capture of tacit knowledge, and organisational is- 
sues surrounding the capture of tacit knowledge are also considered.

\section{Knowledge Management Concepts}

The concept of knowledge management incorporates theory from numerous disciplines. Many definitions exist, yet while there seems to be a lack of consensus about what actually constitutes knowledge management, many definitions recognise the tangible and intangible elements of knowledge. For example, Edwards (1997, p. 29) offers the Gartner Group's definition which states "knowledge management is an integrated, systematic approach to identifying, managing and sharing all of an enterprise's information assets, including databases, documents, policies and procedures, as well as previously unarticulated expertise and experience resident in individual workers." Delphi (1997, p. 12) asserts it encapsulates "the practices and technologies which facilitate the efficient creation and exchange of knowledge on an organization-wide level in order to enhance the quality of decision making." Gladstone (2000, p. 1) is more succinct and concludes "knowledge management is explicitly about how people learn and share together in organisations."

At this point, an attempt to clarify the term "knowledge" is also necessary. Knowledge is defined by Davenport and Prusak (1998, p. 5) as "a fluid mix of framed experience, values, contextual information, and expert insight that provides a framework for evaluating and incorporating new experiences and information." Although debates continue to exist, there seems to be recognition amongst the more familiar knowledge management writers that there is some form of interplay between data, information and knowledge. While data is often recognised as being raw facts and figures, data may be structured in meaningful and purposeful patterns to represent information. Yet the concept of knowledge implies a body of information that is of a higher calibre than information (Terrett, 1998). The common ground seems to be that information in large quantities does not by itself solve business problems, produce value or enhance competitiveness, and that organisational knowledge creation involves a continuous interplay between tacit and explicit knowledge.

Tacit knowledge is linked to personal perspectives, intuition, emotions, beliefs, know-how, experiences and values. It is intangible and not easy to articulate, making it difficult to share with others (Dixon 2000; Inkpen, 1996; Polayni, 1966; Nonaka \& Takeuchi, 1995; von Krogh, Ichijo \& Nonaka 2000). In comparison, explicit knowledge has a tangible dimension that can be more easily captured, codified and communicated. Nonaka and Takeuchi (p. 61) further articulate the distinction between explicit and tacit knowledge and state that when tacit and explicit knowledge interact "knowledge conversion" occurs. They describe the process of creating knowledge as a spiral and believe the interaction of tacit and explicit knowledge produces four modes of knowledge conversion that are interconnected. These modes are socialisation (from tacit to tacit), externalisation (from tacit to explicit), combination (from explicit to explicit), and internalisation (from explicit to tacit).

From a knowledge management perspective, these interrelationships are complex and should not be ignored. In particular, by endeavouring to understand and nurture these relationships an organisation is more likely to succeed with its knowledge management efforts. Yet, whilst organisations aiming to harness their corporate knowledge can be found in all sectors of business, overall the quality of knowledge management efforts continue to be questionable. As an example, Stewart (1997, p. 64) reports Betty Zucker's findings and states that when chief executive officers are asked what percentage of the knowledge in their organisations is used, they typically say "about 20 percent." More recently, outcomes from studies conducted by Kluge, Stein and Licht (2001, p. 89) have a similar flavour and indicate that even though organisations continue to invest heavily in knowledge management "few companies have successfully managed to maximize the opportunities presented by the ability to transfer knowledge." 
Furthermore, the literature also suggests that organisations partaking in knowledge management endeavours continue to concentrate the majority of their efforts in the sharing of explicit rather than tacit knowledge. Indeed, the effectiveness of such pursuits seems problematic. For instance, Stewart (1997, p. 74) convincingly emphasises the need to share tacit knowledge and states that "tacit knowledge needs to become explicit; what's unspoken must be said aloud. Otherwise it cannot be examined, improved or shared." Edwards (1997, p. 31) has similar views and reports that tapping into the knowledge that resides in the heads of employees is vital, especially since, a great proportion of what an organisation should know about its competition is available from its staff, and related service providers. Yet whilst this may be the case, van Ewyk (1998, p. 6) highlights that an individual's knowledge does not belong to his or her employer and at best an organisation rents this knowledge from an employee. Borghoff and Pareschi (1998, p. 6) indicate that organisational tacit knowledge has often been damaged by the introduction of a plenitude of management fads. In many instances, such as those associated with business process reengineering initiatives, the retrenchment of staff has been the result. They argue that many of these workers represent valuable repositories of corporate tacit knowledge. Clearly, from a knowledge management perspective, the risks associated with such initiatives are high and seem to be counterproductive. Effort must therefore be focussed on implementing strategies that aim to capture this valuable know-how during the course of an employee's tenure, for it is likely it will be lost forever when an employee leaves an organisation.

More recently, von Krogh et al (2000, p. 6-7) reinforce the importance and continuing relevance of this argument:

While the idea of tacit knowledge makes sense intuitively to most people, managers often have a hard time coming to grips with it on a practical level. Recognising the value of tacit knowledge and figuring out how to use it is the key challenge in a knowledge-creating company, one that requires extended conversations and good personal relationships - that is knowledge enabling. Tacit knowledge may seem too mysterious to be usefully or consistently applied in a business situation, but this shifting context-specific quality is precisely what makes it a powerful tool for innovation. The challenge comes in enabling such a creative source, rather than ignoring or muzzling it.

\section{Knowledge Management Tools and Technologies}

With this in mind, organisations would thus be served well by introducing processes and technologies that aim to convert tacit knowledge into explicit knowledge and capture it for use by the body corporate. Yet the challenge of exploiting technology for this purpose requires careful consideration of the knowledge management tools and technologies available to meet the needs of the organisation.

Some writers indicate that it is naive to think that there is no distinction between existing technologies and knowledge management solutions, and that knowledge management solutions are merely combinations of existing technologies. For example, Ruggles (1997, p. 3) indicates that true knowledge management tools are not data or information management tools with a contemporary banner. This is because data management tools (eg data warehouses, data search engines, data modelling tools) and information management tools (eg automated search and retrieval agents and document management tools) do not capture the complexity of context and the richness of knowledge and are not robust enough to truly facilitate knowledge management. Delphi (1997) seems to support this view and suggest a knowledge management solution should have the intelligence to automatically infer relationships between different pieces of knowledge, as well as between knowledge, users and processes. Furthermore, Frappaolo and Toms (1997, p. 6) indicate knowledge management solutions should be context sensitive ("understand" the context of the knowledge requirement), user sensitive (organise knowledge in a useful way), flexible (handle knowledge of any form), heuristic (learn from previous queries), and suggestive (deduce knowledge needs). 
Yet, Frappaolo and Toms (1997, p. 4) also acknowledge that many of the knowledge management solutions available on the market tend to consist of combinations and enhancements of existing technologies. They indicate that no single technology will fill all the criteria of a knowledge management system and suggest that the trick is to look "beyond the grand promise of an omnipotent single source solution." Although somewhat contrary to Ruggles views on what constitutes "true knowledge management tools" they advise organisations should not dismiss focussing energy in the creative and strategic deployment of existing technologies to enable their knowledge management efforts.

It seems that specific technologies are likely to play different roles in an organisation's knowledge management endeavours. Existing technologies also have the potential to make a worthwhile contribution. In particular, according to Delphi (1997), the primary technologies for knowledge management include intranets, groupware, search and retrieval engines, databases, and document management systems. Delphi's (p. 18) research also suggests that technology will most strongly contribute in the management of explicit knowledge, "while the role of technology in managing the elusive and valuable tacit knowledge will primarily lie in facilitating face-to-face exchange and knowledge transfer."

\section{Exploring Collaborative Technologies}

However, Davenport and Prusak (1998, p. 81) state "as difficult as it is to codify tacit knowledge, its substantial value makes it worth the effort." In search of capturing and transferring tacit knowledge, the employment of tools and technologies with hypertext and hypermedia capabilities may form part of the solution. For instance, as suggested by Davenport and Prusak, intranets seems to represent an ideal technology for effectively capturing "at least some meaningful fraction of an expert's knowledge, making the tacit explicit." By facilitating access to common repositories of information intranet technologies can readily and inexpensively wire everyone in an organisation to each other. This is especially so because these technologies are easy and intuitive to use and are flexible enough to be adapted to changing environments. Such infrastructures provide an ideal environment for sharing of information that is both dynamic and richly linked, can be used for asynchronous communication across different time zones (eg electronic discussion groups), and can enhance communication and collaboration (Dataware Technologies, 1998; Cohen, 1998). An intranet is also valuable for use in teleworking and virtual team settings since it can allow knowledge from individuals to be transferred into a central repository and thus facilitate the exchange of, for example, insights and experiences (Davenport, De Long \& Beers, 1998).

Essentially, as highlighted by Scott (1998, p. 3) "possibly the most far-reaching impact of Intranet use is on organisational knowledge creation." Scott uses Nonaka and Takeuchi's theory to describe how. As mentioned previously, this knowledge creation theory hinges on the distinction between tacit and explicit knowledge and incorporates the four modes of knowledge conversion (shown in Table 1), depicting a life cycle of organisational knowledge creation. 


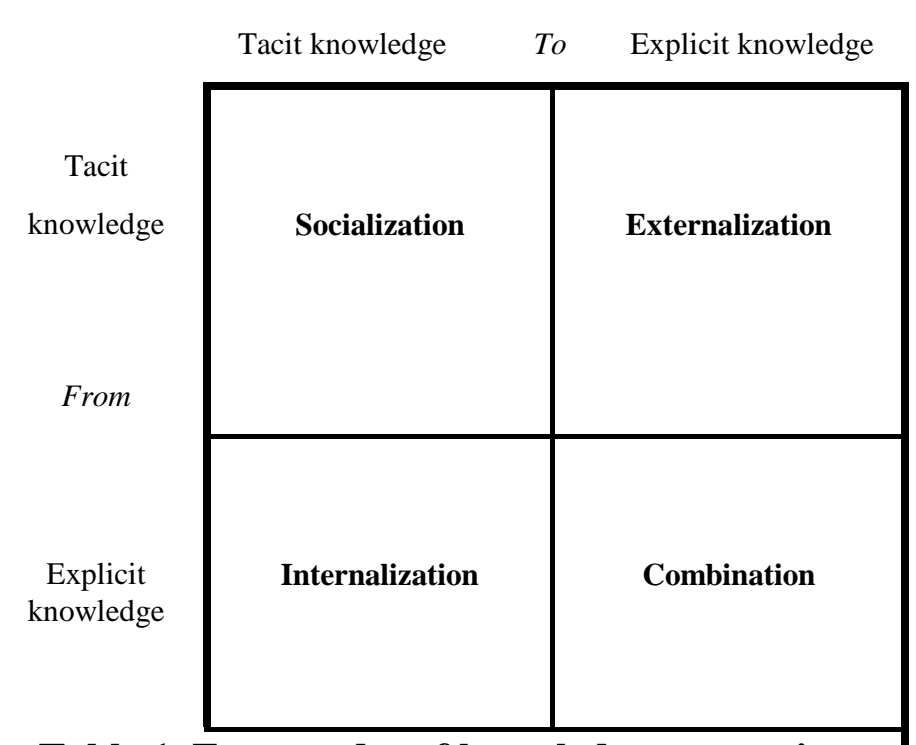

Table 1. Four modes of knowledge conversion (from Nonaka and Takeuchi, 1995, p. 62)
Scott (p. 8) indicates an intranet can help facilitate socialisation by incorporating multimedia such as animation, graphics, audio and virtual reality. This can be achieved by the use of video clips that demonstrate operational procedures and methods and is particularly useful when procedures cannot be easily communicated or when language barriers exists. In relation to externalisation, Scott (p. 9) identifies the hyperlinking capability as a means of reducing cognitive overload and highlights graphics "can enhance the use of metaphors, analogies and prototypes to clarify what was originally fuzzy and obscure." In the process of facilitating internalisation examples such as spreadsheet-like 'what if' scenarios and simulations incorporated into intranet decision support applications are offered. Additionally, Scott cites that the combination mode is facilitated through the application of "categorization and information processing."

The hyperlinking capability also has special significance in other knowledge management activities aimed towards the capture of tacit knowledge. Gundry and Metes (1996, p. 14) believe "the answer to the restructuring of knowledge in computer conference records is hyperlinking." They emphasise the importance and value of sharing context, because it is context that assists in enabling the tacit dimension to be communicated via the emergence of explicit relationships. By turning the content of a computer conference into a "web of knowledge", computer conference records can be made accessible to a variety of future users enabling viewpoints to be shared and reused. For example, by accessing such records, an individual that wishes to understand how a team dealt with a particular problem situation, is more likely to comprehend the rationale surrounding decisions that were made. Yet as noted by Gundry and Metes ( $\mathrm{p}$. 15), an important task in employing hyperlinking in this way is to "identify the substantive items of content in computer conference records, and link them together." Essentially, by preserving these relationships, hyperlinking can help to enable a shared context for a particular subject matter, and assist in facilitating the conversion of individual tacit knowledge to explicit knowledge. In support of this, Fahey and Prusak (1998, p. 268) suggest that a shared context is important since in its absence, "individuals' differing perspectives, beliefs, assumptions, and views of the future are most likely to collide and thus immobilise decision-making."

Along with hyperlinking, according to Shum (1999, p. 8), "hypermedia is an ideal technology for capturing informal knowledge types with relationships that are hard to formalise." The use of hypermedia infrastructures can promote collaboration and shared understanding and facilitate the capture of tacit knowledge, which in turn contributes to organisational learning and organisational memory. Once again, the idea of context sharing is extremely pertinent. As explained by Shum (p. 9) outcomes from meetings and debates do not exist in a vacuum. These outcomes are often related to existing and future work that is based on and generates a variety of interrelated and relevant artefacts such as email correspondence, financial information, business cases and graphical representations. The use of hypermedia technology can enable the richness of this type of media to be adequately stored, integrated and retrieved. Ultimately, this contributes to the sharing of tacit knowledge and increases the possibility of the intangible becoming tangible 
through the explicit sharing of context relating to a particular decision making environment or problem situation.

In particular, the use of hypermedia groupware systems such as QuestMap can enable the preservation and sharing of such connections and context. Unlike traditional groupware such as e-mail, QuestMap provides a display system that graphically maps conversations to show how comments, issues, arguments and artefacts pertaining to a particular problem situation are related. As noted by Eppel and Conklin (1999), the chronological view typical of groupware such as e-mail omits the richness of context and thus the possi-

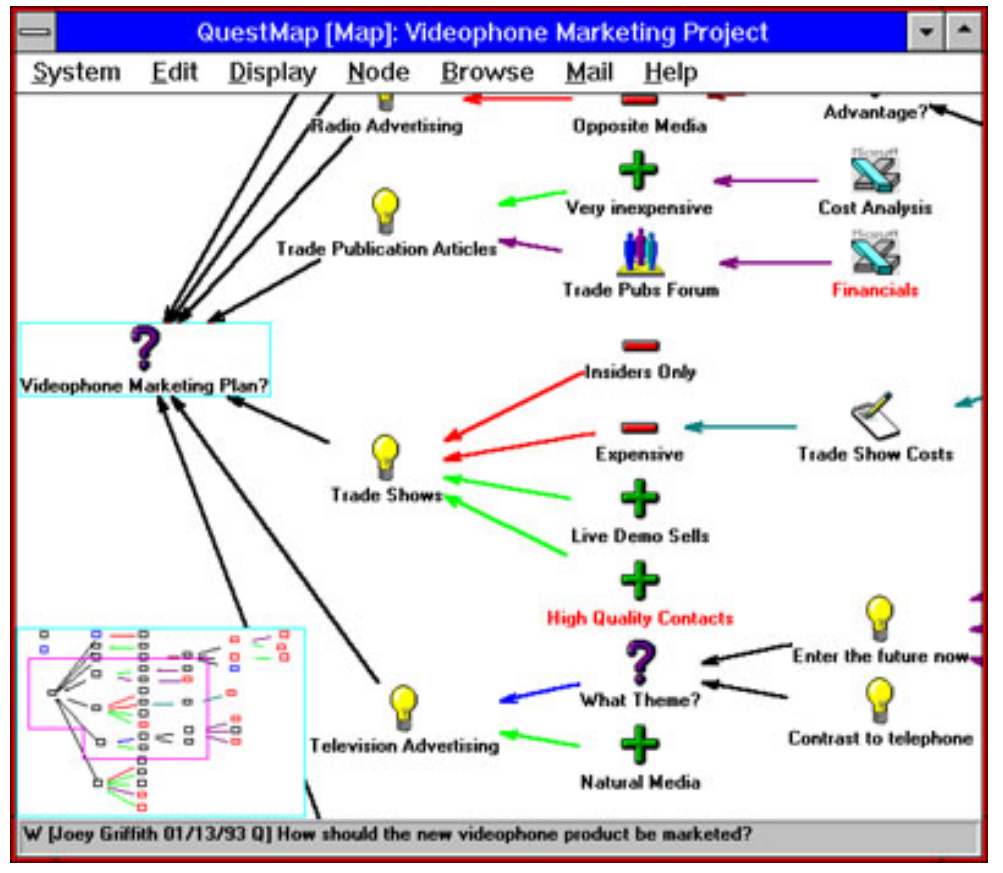

Figure 1. example of an enquiry (Source: Conklin, 1996, p. 19)

bility for misinterpretation and misunderstanding is increased. The following screen (Figure 1) shows an example of an enquiry that was developed using the QuestMap software.

Essentially, QuestMap enables the capture of an explicit representation and electronic memory of conversations and related artefacts. Accordingly, hypermedia groupware systems such as QuestMap are also said to be useful in supporting the taming of wicked problems. Wicked problems are difficult to solve, generally lack a definitive statement of the problem, have changing constraints, involve many stakeholders, require iteration, and are usually so complex that the problem is not understood until a solution (there is no definitive solution) has been developed (Conklin \& Weil, 1999; Shum, 1999). In the process of solving such problems a broad range of thinking and the reliance on expert knowledge is often evident. With the use of QuestMap problem solvers can electronically capture the relationships between the diverse sets of ideas that are generated. These explicit representations help with the process of reflecting on the various arguments that are posed during the duration of the decision making process. Additionally, as suggested by Conklin (1999), as conversations are captured the organisation begins to accrete a memory of informal knowledge, linked within itself, as well as with any related documents that are on the corporate network.

\section{Organisational Considerations and Implications}

With the aim of effectively capturing a meaningful fraction of an expert's tacit knowledge to make it explicit, the use of technologies with hyperlinking and hypermedia capabilities seem viable. However, whilst such technology may help to enable an organisation's knowledge management efforts, the technology should not be considered in isolation, but instead as a part of the total organisational environment. The challenge, therefore, of enabling effective knowledge management outcomes goes beyond merely a technology-centred approach.

Von Krogh et al (2000, p. 28) discuss the importance of employing a balanced approach when facilitating knowledge management initiatives. In particular, they highlight that organisations must be aware that an overemphasis on correctly applying the technology can sometimes end up "outweighing the results of knowledge creation." This view is supported by Davenport $(1997$, p. 1) who indicates if an organisation is 
spending more than one-third of its time on technologies for knowledge management it is "neglecting the content, organisational culture and motivational approaches that will make a knowledge management system actually useful." Delphi (1997) also highlight that technology is only one part of the solution, and indicate knowledge management solutions should encapsulate four key components. These are business strategy, corporate culture, information management, and technology. Of these, support from the corporate culture is identified as essential when implementing effective knowledge management systems (Conklin 1999; Dataware Technologies 1998, Davenport et al 1998; Edwards 1997; Terrett 1998).

Reporting the findings of the "McKinsey \& Company global survey on knowledge management" Kluge, Stein and Licht (2001, p. 25) reflect on their experiences and state:

Many companies we visited had already tried to introduce knowledge management programs, and at times these efforts were quite substantial. But despite management commitment and healthy budgets, these programs often floundered or failed. In each case something was missing. A vital ingredient of the knowledge management recipe had not just been left in the cupboard; it was not even on the shopping list. All these companies lacked the right cultural context that would create and nurture reciprocal trust, openness and cooperation.

Indeed, there can be little doubt that creating a culture that encourages the sharing of tacit knowledge is imperative. Yet, this type of knowledge management effort also relies on active participation, and commitment from all involved. As suggested by Shum (1999, p.16), the successful implementation of collaborative technologies requires the adoption of a "relatively open, transparent mode of communication, negotiation and accountability." In particular, people with influence must be actively involved if an organisation is going to attempt to capitalise on tacit knowledge creation technologies and processes. Edwards (1997, p. 32) identifies top management support as "a key differentiator in the speed and success of implementation of a knowledge management program." Inkpen (1996) also emphasises the need and importance of the explicit commitment of organisational leaders. Scott (1998, p. 14) identifies that top management can greatly influence corporate knowledge management efforts by ensuring funds are available to implement and maintain the technology; recruiting appropriate technical expertise; offering incentives; encouraging socialisation; and by nurturing a culture that respects the value and need for the articulation of knowledge. Additionally, knowledge creation efforts surrounding the use of hypermedia technologies would require managers to be tolerant of the concept of information "redundancy", which according to Inkpen (1996) arises when issues are debated and assumptions are questioned. For without such tolerance, difficulty would be experienced in maintaining the flow of dialogue and the continued sharing of ideas that knowledge creation efforts require.

In the absence of an appropriate culture and management commitment, the challenges associated with implementing such technologies should not be underestimated. The introduction of technologies with hyperlinking and hypermedia capabilities is likely to change organisational patterns of access to information and knowledge. This may be threatening in situations where hoarding of information is prevalent and where there is a climate typified by the notion that knowledge represents power. Yet according to Corrall (1999, p. 3), the technology "has the potential to change culture by cutting through traditional structures, inspiring an informal style and fostering the social networks which underpin knowledge-sharing." The Lotus Development Corporation (1998) further indicates that its real-world experience suggests that the technology can overcome some of the "cultural disinclination towards knowledge management" and the key is to design the technology to "overcome - or at least offset - these human barriers." 


\section{Conclusion}

In the business environments of today, the ability to create, transfer and use knowledge effectively is becoming an essential prerequisite to success. Knowledge is increasingly described as an asset and an essential source of competitive advantage. The theory of organisational knowledge creation suggests the sharing of tacit knowledge is a critical component of successful knowledge management efforts. Accordingly, to survive in this emerging economy of knowledge, organisations must recognise the need to introduce processes and technologies that aim to convert tacit knowledge into explicit knowledge and then capture it for use by the body corporate.

To effectively enable the capture of a meaningful fraction of an expert's tacit knowledge and make it explicit, it seems clear that there is value in employing the use of technologies with hyperlinking and hypermedia capabilities. However, the challenge of exploiting this type of technology requires the application of a holistic mindset. This not only includes careful consideration of the various knowledge management tools and technologies available to meet the needs of the organisation, but also careful consideration of the related management and cultural mix that is required to facilitate such endeavours.

The technologies described in this paper are highly collaborative in nature. To successfully implement such technologies an organisation must be committed to actively nurture a corporate culture that is supportive of the sharing of knowledge across traditional organisational boundaries.

\section{References}

Borghoff, U., \& Pareschi, R. (Eds.). (1998). Information technology for knowledge management._New York: Springer.

Cohen, S. (1998). Knowledge management's killer app. Training and Development, 52(1), 50-57.

Conklin, J. (1996). Designing organisational memory: Preserving intellectual assets in a knowledge economy. Retrieved June 9, 2001, from the Group Decision Support Systems Web site: http://www.gdss.com/wp/DOM.htm

Conklin J., \& Weil W. (1999). Wicked problems: Naming the pain in organisations. Retrieved September 31, 2001, from the Group Decision Support Systems Web site: http://www.gdss.com/wp/wicked.htm

Corrall, S. (1999). Knowledge management: Are we in the knowledge management business? Knowledge Management, 18. Retrieved May 26, 2001, from http://www.ariadne.ac.uk/issue18/knowledge-mgt/

Dataware Technologies. (1998). Knowledge management: Linking people to knowledge for bottom line results, Retrieved March 22, 1999, from http://www.dataware.com

Davenport, T. (1997). Known evils: Common pitfalls of knowledge management. Retrieved 29 March 29, 1999, from http://www.cio.com/archive/061597_think_print.html

Davenport, T., De Long, D., \& Beers, M. (1998). Successful knowledge management projects. Sloan Management Review, $39(2), 43-57$.

Davenport, T., \& Prusak L. (1998). Working knowledge: How organisations manage what they know. Boston: Harvard Business School Press.

Delphi Group. (1997). Delphi on Knowledge Management.

Dixon. (2000). Common knowledge: How companies thrive by sharing what they know. Boston: Harvard Business School Press.

Edwards, L. (1997). White Paper: Managing knowledge for competitive advantage. KnowledgeX, Course 101 Readings.

Eppel R., \& Conklin J. (1999). Blending cultural transformation and groupware to create a learning organisation. Retrieved October 31, 2001, from http://www.gdss.com/learning.htm

Fahey, L., \& Prusak, L. (1998) The eleven deadliest sins of knowledge management, California Management Review, 40(3), 265-276.

Frappaolo, C., \& Toms, W. (1997). From Terra Incognita to Terra Firma. The Delphi Group, Course 101 Readings. 
Gladstone, B. (2000). From Know-how to knowledge: The essential guide to understanding and implementing knowledge management. London: The Industrial Society.

Gundry, J., \& Metes G. (1996). Team knowledge management: A computer-mediated approach. A working by wire white paper from Knowledge Ability Ltd, Malmesbury UK and Virtual Learning Systems, Inc., Manchester NH USA, Retrieved November 22, 2001, http://www.knowab.co.uk/wbwteam

Inkpen, A. (1996). Creating knowledge through collaboration, California Management Review, 39(1), 123-140.

Kluge, J., Stein, W., \& Licht, T. (2001) Knowledge unplugged: The McKinsey \& Company global survey on knowledge management. Houndmills: Palgrave.

Lotus Development Corporation. (1998). White Paper: Lotus, IBM, and Knowledge Management” Retrieved March 22, 1999, from http://www.lotus.com/news/topstories.nsf/

Nonaka, I., \& Takeuchi, H. (1995). The knowledge creating company: How Japanese companies create the dynamics of innovation. New York: Oxford University Press.

Polayni, M. (1966). The tacit dimension. London: Routledge and Kegan Paul.

Ruggles, R., III, (Ed.). (1997). Knowledge management tools. Newton: Butterworth-Heinemann.

Scott, J. (1998). Organisational knowledge and the intranet. Decision Support Systems, 23, 3-17.

Shum, S. (1998). Negotiating the construction of organisational memories. Retrieved May 25, 2001 from http://d3e.open.ac.uk/general/negotiating-org-mem/negotiating-org-mem-01.html

Stewart, T. (1997). Intellectual capital: The new wealth of organisations. New York: Doubleday.

Terrett, A. (1998). Knowledge management and the law firm. Journal of Knowledge Management, 2(1), 67-76.

van Ewyk, O. (1998). Preparing for the knowledge era, Retrieved March 22, 2001, from http://www.hci.com.au/hcisite/articles/prepare.htm

von Krogh, G., Ichijo, K., \& Nonaka, I. (2000). Enabling knowledge creation: How to unlock the mystery of tacit knowledge and release the power of innovation New York: Oxford University Press.

Wiig, K. (1997). Knowledge management: Where did it come from and where will it go? Expert Systems with Applications, 13(1), 1-14.

\section{Biography}

Vicki Romaldi is currently employed as an information systems practitioner in the South Australian public sector. Previously, she has held a number of management positions where she has managed a diverse range of strategic management and continuous improvement initiatives. She is also a PhD candidate and Research Associate at the University of South Australia's Information Systems Doctoral School. She holds a Bachelor of Business in Management Information Systems from the University of South Australia, and postgraduate qualifications in Management from the University of Western Sydney. Her research interests are in the areas of strategic information systems, group support systems and knowledge management. 